\title{
Enhanced catalytic performance of Zncontaining HZSM5 upon selective desilication in ethane dehydroaromatization process
}

Goodarzi, Farnoosh; Pulikkal Thumbayil, Rouzana; Enemark-Rasmussen, Kasper; Mielby, Jerrik Jørgen; Nguyen, Thoa T.M.; Beato, Pablo; Joensen, Finn; Kegnæs, Søren

\section{Published in:}

ChemCatChem

Link to article, DOI:

$10.1002 /$ cctc. 201902123

Publication date:

2020

Document Version

Peer reviewed version

Link back to DTU Orbit

Citation (APA):

Goodarzi, F., Pulikkal Thumbayil, R., Enemark-Rasmussen, K., Mielby, J. J., Nguyen, T. T. M., Beato, P., Joensen, F., \& Kegnæs, S. (2020). Enhanced catalytic performance of Zncontaining HZSM5 upon selective desilication in ethane dehydroaromatization process. ChemCatChem, 12(5), 1519-1526. https://doi.org/10.1002/cctc.201902123

\section{General rights}

Copyright and moral rights for the publications made accessible in the public portal are retained by the authors and/or other copyright owners and it is a condition of accessing publications that users recognise and abide by the legal requirements associated with these rights.

- Users may download and print one copy of any publication from the public portal for the purpose of private study or research.

- You may not further distribute the material or use it for any profit-making activity or commercial gain

- You may freely distribute the URL identifying the publication in the public portal 


\section{Heterogeneous \& Homogeneous \& Bio- \& Nano-}

\section{CATALYSIS}

\section{Accepted Article}

Title: Enhanced catalytic performance of Zn-containing HZSM-5 upon selective desilication in ethane dehydroaromatization process

Authors: Farnoosh Goodarzi, Rouzana Pulikkal Thumbayil, Kasper Enemark-Rasmussen, Jerrik Mielby, Thoa T.M. Nguyen, Pablo Beato, Finn Joensen, and Soeren Kegnaes

This manuscript has been accepted after peer review and appears as an Accepted Article online prior to editing, proofing, and formal publication of the final Version of Record (VoR). This work is currently citable by using the Digital Object Identifier (DOI) given below. The VoR will be published online in Early View as soon as possible and may be different to this Accepted Article as a result of editing. Readers should obtain the VoR from the journal website shown below when it is published to ensure accuracy of information. The authors are responsible for the content of this Accepted Article.

To be cited as: ChemCatChem 10.1002/cctc.201902123

Link to VoR: http://dx.doi.org/10.1002/cctc.201902123 


\title{
Enhanced catalytic performance of Zn-containing HZSM-5 upon selective desilication in ethane dehydroaromatization process
}

\author{
Farnoosh Goodarzi, ${ }^{[a]}$ Rouzana Pulikkal Thumbayil, ${ }^{[a]}$ Kasper Enemark-Rasmussen, ${ }^{[a]}$ Jerrik Mielby, \\ [a] Thoa T.M. Nguyen, ${ }^{[b]}$ Pablo Beato, ${ }^{[b]}$ Finn Joensen ${ }^{[b]}$ and Søren Kegnæs ${ }^{*[a]}$
}

\begin{abstract}
The effect of introducing mesoporosity on the catalytic stability and selectivity of Zn-containing ZSM-5 catalysts was investigated for direct conversion of ethane to high-value aromatics. Voids and mesopores were created in the zeolite $(\mathrm{Si} / \mathrm{Al}=40)$ by selective desilication (recrystallization) using ammonium hydroxide and cetyltrimethylammonium bromide. Zinc was incorporated in the samples by incipient wetness impregnation to achieve different concentrations of $0.5,1$ and $5 \mathrm{wt} \%$. The samples were characterized by XRD, $\mathrm{N}_{2}$ physisorption, TGA, $\mathrm{NH}_{3}$-TPD, ICP-OES, FTIR, ${ }^{1} \mathrm{H},{ }^{27} \mathrm{Al}$ and ${ }^{29} \mathrm{Si}$ solid-state MAS NMR. The desilicated samples were compared to their parent analogues for ethane conversion to aromatics such as benzene, toluene and xylenes (BTX). All of the desilicated samples showed an improvement in BTX yield and catalytic stability. Thus, the presence of mesoporosity increases the accessibility to the active sites and facilitates the mass transfer of aromatic compounds, which result in higher performance and catalytic stability of the desilicated ZSM- 5 samples containing zinc.
\end{abstract}

\section{Introduction}

Benzene, toluene, and xylenes (BTX) have extensive applications as basic raw materials for many industrial chemicals (e.g. production of styrene and phenol) and gasoline blendstocks. ${ }^{[1-3]}$ Current industrial production of BTX involves naphtha reforming using crude oil as the starting material. ${ }^{[4]}$ Replacing crude oil with an economic and abundant feedstock is highly desired to reduce the environmental footprint of aromatic synthesis. With the low price of natural gas - and even declining over time -, natural gas opens up an opportunity to produce BTX from the light hydrocarbon components of natural gas. Considering that ethane is often an abundant component in natural gas, the process for the direct conversion of ethane to BTX has gained increased attention in the industry for the last few decades. ${ }^{[5-8]}$ Hence, this work is focused on studying the direct conversion of ethane into BTX.

[a] F. Goodarzi, R. P. Thumbayil, Dr. K.E. Rasmussen, Dr. J.Mielby and Prof. S. Kegnæs

DTU Chemistry

Technical University of Denmark

Kemitorvet 207, DK-2800 Kgs. Lyngby, Denmark

E-mail: skk@kemi.dtu.dk

[b] Dr. P. Beato, Dr. T. T. M. Nguyen, F. Joensen Haldor Topsoe A/S

Haldor Topsøes Allé 1, DK-2800 Kgs. Lyngby, Denmark

Supporting information for this article is given via a link at the end of the document.
ZSM-5 is a well-known zeolite with solid acid sites that has been developed for and is widely used in chemical and petroleum industrial processes such as isomerization, alkylation and hydrocracking. ${ }^{[9,10]}$ The strong acid sites make ZSM-5 a promising candidate for aromatization of olefins. ${ }^{[11,12]}$ Another advantage of ZSM-5 zeolite is its thermal stability and unique pore structure. The pore channels are about the size of para-xylene, which provides a unique shape selectivity. ${ }^{[13,14]}$

In order to directly convert alkanes to aromatics, a bi-functional catalyst is required, which in addition to the acid function comprises the dehydrogenation functionality of metal such as $\mathrm{Ga}$, $\mathrm{Zn}, \mathrm{Mo}, \mathrm{Re}$ or $\mathrm{Pt}^{\left[{ }^{15-23]}\right.}$ Numerous studies demonstrated that incorporation of Lewis acid metals such as $\mathrm{Zn}$ into zeolites modifies the surface acidity and enhances selectivity to BTX in light alkane dehydromatization. ${ }^{[7,15,18,24-27]}$ However, only a few studies have been dedicated to the performance and catalytic lifetime enhancement of $\mathrm{Zn}$-containing zeolite catalyst for ethane direct conversion to aromatics. Vosmerikova et al. ${ }^{[28]}$ studied the influence of zeolite framework structure for ethane dehydroaromatization on ZSM-5, ZSM-8, ZSM-11, and ZSM12 at $650^{\circ} \mathrm{C}$. They demonstrated that the most effective catalyst is zinc containing ZSM-5. Mehdad et al. ${ }^{[6]}$ investigated the conversion of ethane and ethylene on zinc-containing SSZ-13 and ZSM-5. They showed that $\mathrm{Zn} / \mathrm{ZSM}-5$ had the highest activity for ethane conversion at $500{ }^{\circ} \mathrm{C}$ over $5 \mathrm{~h}$ operation time. In addition, they concluded that zinc in the Lewis acid site position can perform aromatization while small $\mathrm{ZnO}$ species can only catalyze dehydrogenation reactions. Liang et al. ${ }^{[24]}$ studied the activation energy for ethane and ethylene aromatization via transient kinetic methods using zinc exchanged HZSM-5. Their study showed a higher activation energy for ethane aromatization when compared with ethylene. Robinson et al. ${ }^{[29]}$ promoted Mo/ZSM-5 catalyst with zinc for ethane dehydroaromatization, which resulted in higher aromatic selectivity at $615^{\circ} \mathrm{C}$ during 21 min reaction.

In general, deactivation of ZSM-5 catalysts due to coking is an issue in ethane dehydroaromatization like in many other hydrocarbon conversion processes. The high density of Brønsted acid sites present in ZSM-5 zeolites can accelerate carbon deposition. ${ }^{[30]}$ It has been demonstrated that adding zinc to ZSM5 decreases the density of Brønsted acid sites ${ }^{[3]}$ and therefore, coking rate is decreased. Saito et al. ${ }^{[31]}$ also improved the catalytic life-time of Zn/ZSM-5 catalyst by performing steam treatment. They showed that preferential dealumination resulted in coke suppression by partial removal of Brønsted acid sites. However, the imposed diffusion barrier in ZSM-5 due to the medium size of the micropores has been a challenge for bulky molecules with the size similar or larger to the pore size of the MFI structure. Thus, mass transfer limitation can be another cause of coke deposition besides affecting the conversion and selectivity. ${ }^{[32]}$ Enhancing 
mass transfer could increase accessibility to the active sites and facilitate transfer of molecules out of the crystal, thereby inhibiting further conversion of desired products into coke species. Nowadays, the leading materials to improve catalytic performance by minimizing the mass transfer limitation are hierarchical zeolites consisting of both micro and mesopores that facilitate transport of bulky molecules. ${ }^{[33-35]}$ In general, there are two approaches to synthesis hierarchical zeolites: either by direct synthesis using hard/soft templates or post-modification such as alkali/acid treatment. ${ }^{[36,37]}$ Han et al. ${ }^{[38]}$ successfully dissolved and reassembled the ZSM-5 crystals in the presence of a surfactant via a simple and efficient method to create mesoporosity by removing the silicon (desilication) without destroying the zeolite structure. This approach was applied later in other studies to achieve hierarchical zeolite structures. ${ }^{[39,40]}$

Building on these previous results, the present study investigates the effect of creating mesopores using ammonium hydroxide and surfactant for selective desilication in a HZSM-5 sample ( $\mathrm{Si} / \mathrm{Al}$ ratio 40$)$ after introducing three different zinc loadings $(0.5,1$ and 5 wt $\%)$. The materials were characterized using different techniques including X-ray diffraction, electron microscopy, infrared spectroscopy and solid-state NMR. Ethane conversion, BTX selectivity and life-time of the modified samples were compared to their parent zeolites. It was demonstrated that the introduced mesoporosity enhances the catalytic performance and life-time of the catalysts. Furthermore, the mesoporous catalyst with the highest zinc loading was the preferred catalyst due to its higher conversion, BTX yield and stability. The conversion of ethane and yield of BTX for this catalyst was $40 \%$ and $22 \%$, respectively, at $550^{\circ} \mathrm{C}$ and 2 barg.

\section{Results and Discussion}

Conventional and desilicated HZSM-5 with Si/Al ratio of 40 were synthesized as described in the experimental section and designated as C-ZSM-5 and D-ZSM-5, respectively. Afterwards, the synthesized zeolites were impregnated with zinc nitrate until incipient wetness to achieve $\mathrm{Zn}$ loadings of $0.5,1$ and $5 \mathrm{wt} \%$ followed by calcination in the reactor at $550{ }^{\circ} \mathrm{C}$ (see catalytic test section). The zinc-containing C-ZSM-5 and D-ZSM-5 were designated as C-ZSM-5-X\%Zn and D-ZSM-5-X\%Zn, where $X=0.5,1$ and 5 for $0.5 \mathrm{wt} \%, 1 \mathrm{wt} \%$ and $5 \mathrm{wt} \% \mathrm{Zn}$ loading, respectively.

Various techniques characterized the prepared samples. The XRD patterns of the fresh catalysts (after calcination in air and before ethane conversion) are compared in Figure $1 \mathrm{~A}$ and B. All conventional and desilicated samples present the characteristic diffraction pattern of the MFI structure and crystallinity is preserved in all of the samples containing zinc. ${ }^{[41]}$ Besides a weak background signal appearing in the desilicated ZSM- 5 samples $\left(12-22^{\circ}\right.$ range), there is no significant contribution of amorphous phases detected. Moreover, $\mathrm{ZnO}$ diffraction peaks at $31.8^{\circ}$ and $36.3^{\circ}$ are detected in the diffraction patterns of C-ZSM- 5 and DZSM- 5 samples containing $5 \mathrm{wt} \%$ zinc. This proves the presence of some $\mathrm{ZnO}$ crystals in these samples, large enough in size to contribute to the diffraction pattern.

The $\mathrm{N}_{2}$ physisorption isotherms for the parent C-ZSM- 5 and DZSM-5 are presented in Figure1C. Both parent ZSM-5 samples exhibit typical type I isotherms. There is a small hysteresis loop at around $P / P_{0}=0.15$ in D-ZSM- 5 sample. This characteristic loop is well-known to appear for $\mathrm{N}_{2}$ physisorption in microporous MFI structure and are not evidence of mesopores but related to a phase transition of the adsorbed $\mathrm{N}_{2}$ from disordered to an ordered phase. ${ }^{[42,43]}$ The BJH analysis of the desorption branch results in a sharp peak at $4 \mathrm{~nm}$ (Figure1D), which is an artefact of the BJH analysis caused by the well-known tensile strength effect (TSE) rather than indicating mesopores. Moreover, another broad hysteresis loop from $P / P_{0}=0.4-1.0$, is present for the desilicated ZSM-5 (type H4). This type H4 loop is ascribed to intraparticle mesopores. Furthermore, BJH analysis for D-ZSM-5 results in a broad pore size distribution from 20-100 nm compared to C-ZSM5 , which causes increased total pore volume and external surface area.
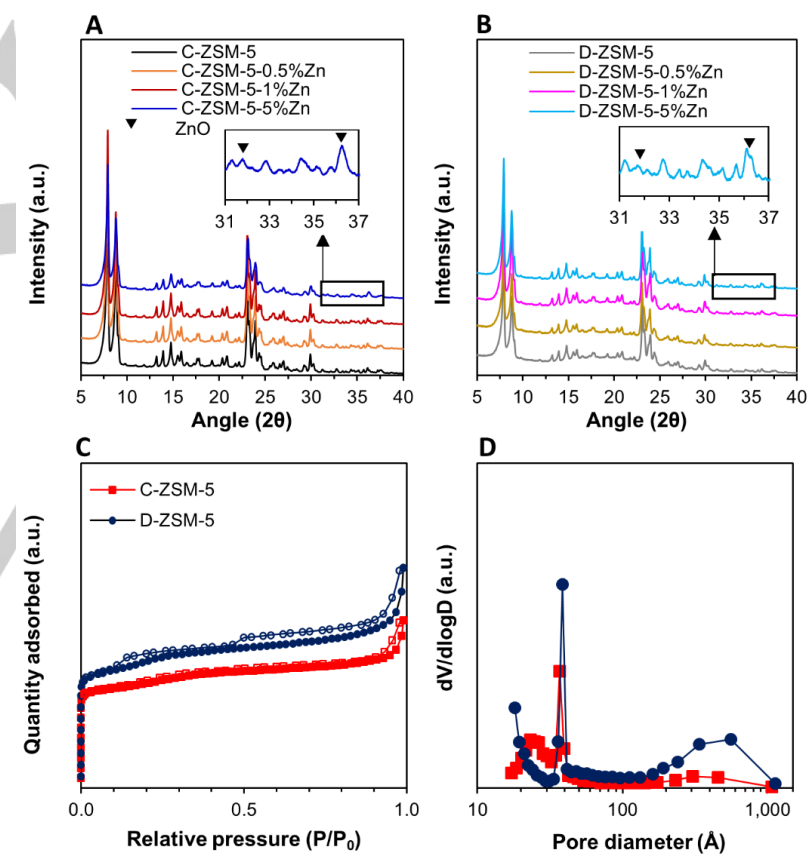

Figure 1. A) \& B) XRD patterns of C-ZSM-5 and D-ZSM-5 zeolites with $0,0.5$ 1 and $5 \mathrm{wt} \%$ zinc content after impregnation and calcination at $550{ }^{\circ} \mathrm{C}$ for $2 \mathrm{~h}$, C) Nitrogen adsorption-desorption isotherms and D) Pore diameter distribution obtained from BJH analysis on desorption branch for the fresh C-ZSM-5 and DZSM.

The textural properties of parent C-ZSM- 5 and D-ZSM- 5 samples including the BET surface area, total pore volume and micropores volume are obtained from $\mathrm{N}_{2}$ physisorption and summarized in Table 1. The total pore volume is higher in D-ZSM- compared to the C-ZSM-5 sample. Furthermore, micropore volume decreases in D-ZSM- 5 as a result of desilication and formation of mesopores at the expense of micropores. Thus, D-ZSM- 5 zeolite provides a 
higher surface and accessibility to the active sites for the catalytic reaction in the zinc-containing catalysts prepared from D-ZSM-5.

Table 1. Textural properties of parent zeolites.

\begin{tabular}{llll}
\hline Sample & $\mathrm{S}_{\mathrm{BET}^{\mathrm{a}}}$ & $\mathrm{V}_{\text {micro }}\left(\mathbf{c m}^{3} / \mathbf{g}\right)^{\mathrm{b}}$ & $\mathrm{V}_{\text {total }}\left(\mathrm{cm}^{3} / \mathbf{g}\right)^{\mathrm{c}}$ \\
\hline C-ZSM-5 & 380 & 0.116 & 0.199 \\
D-ZSM-5 & 424 & 0.108 & 0.251 \\
\hline
\end{tabular}

a Calculated using BET method, ${ }^{\mathrm{b}}$ Calculated using $t$-plot, ${ }^{\mathrm{c}}$ Total pore volume up to $P / P_{0}=0.95$.

Figure 2 shows the TEM images of the conventional and desilicated ZSM-5. It is clear that both C-ZSM-5 and D-ZSM-5 have similar uniform crystal size ( 200-250 nm) and morphology. SEM images of these samples provided in the supporting information (Figure S1), confirm that the TEM images are representative. Mesopores of irregular shape and broad size distribution between 20-50 nm, are observable only for D-ZSM-5, in agreement with the physisorption results and confirming the hierarchical porosity of this sample compared to the purely microporous C-ZSM-5.
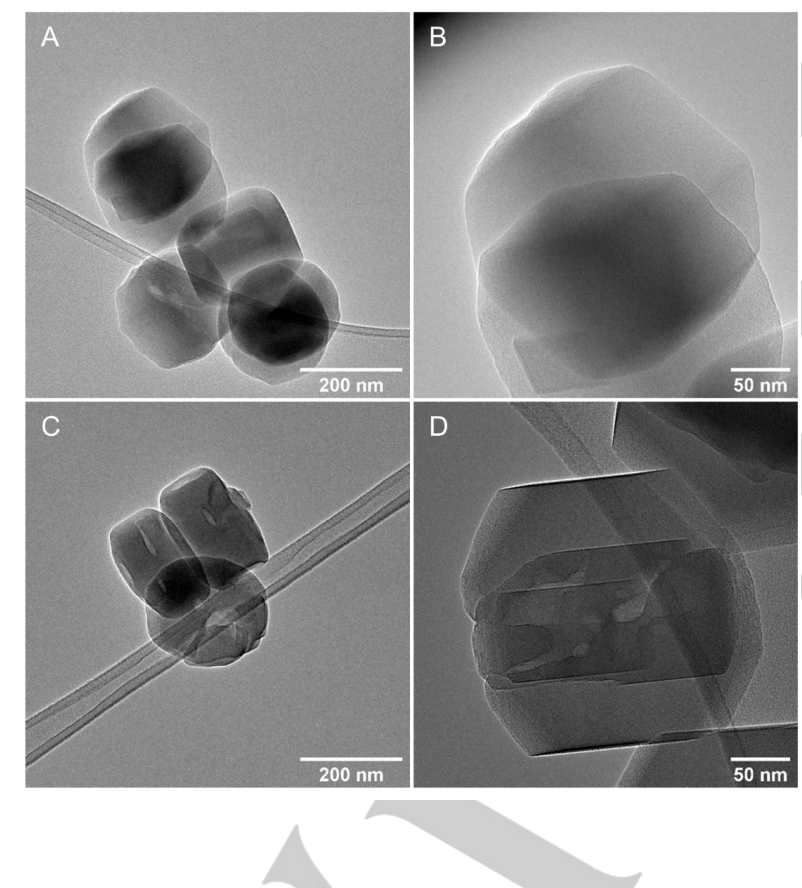

$\overline{50 \mathrm{~nm}}$

Figure 2. TEM images of C-ZSM-5 (A\&B) and D-ZSM-5 (C\&D) zeolites.

Additionally, reported in Table 2 are the results of ICP analysis, comparing the Si/Al molar ratio of the parent conventional and desilicated ZSM-5. As the results show, Si/AI molar ratio slightly decreases from the initial ratio of 42 to 38 after the desilication caused by removal of silicon. Furthermore, Table 2 reports the acidic properties for different zinc-containing samples. As expected from previous investigations ${ }^{[3,7,15]}$, we notice an increase in density of weak acid sites and decrease in strong acid sites, probably caused by coordinative unsaturated $\mathrm{Zn}^{2+}$ species as the zinc loading increases.

Table 2. Metal content and acidic properties of ZSM-5 and Zn-containing ZSM5 samples.

\begin{tabular}{lccccc}
\hline Catalyst & \multicolumn{2}{c}{ Zn wt\% (ICP) } & \multicolumn{2}{c}{$\begin{array}{c}\text { Acidity by strength } \\
\text { (mmol/g) }\end{array}$} \\
\cline { 2 - 6 } & Fresh & Retained $^{\text {a }}$ & Strong $^{\text {b }}$ & Weak $^{c}$ Total \\
\hline C-ZSM-5* & - & - & 0.137 & 0.038 & 0.136 \\
C-ZSM-5-0.5\%Zn & 0.43 & 0.39 & 0.098 & 0.045 & 0.175 \\
C-ZSM-5-1\%Zn & 0.97 & 0.61 & 0.088 & 0.051 & 0.143 \\
C-ZSM-5-5\%Zn & 4.72 & 1.59 & 0.024 & 0.112 & 0.139 \\
D-ZSM-5\# & - & - & 0.166 & 0.068 & 0.173 \\
D-ZSM-5-0.5\%Zn & 0.51 & 0.48 & 0.132 & 0.069 & 0.234 \\
D-ZSM-5-1\%Zn & 0.91 & 0.69 & 0.099 & 0.080 & 0.201 \\
\hline D-ZSM-5-5\%Zn & 4.91 & 1.68 & 0.053 & 0.120 & 0.179 \\
\hline
\end{tabular}

* $\mathrm{Si} / \mathrm{Al}=42$ and 39 obtained from ICP-OES and MAS NMR, respectively, \# $\mathrm{Si} / \mathrm{Al}=38$ and 35 obtained from ICP-OES and MAS NMR, respectively. ${ }^{a}$ Retained $\mathrm{Zn}$ is the remaining $w \mathrm{t} \% \mathrm{Zn}$ in the catalyst after reaction for $30 \mathrm{~h}$ at $550{ }^{\circ} \mathrm{C}$., b ${ }^{b}$ Density of acid sites determined by $\mathrm{NH}_{3}$-TPD for $\mathrm{NH}_{3}$ desorbed at 280-550 ${ }^{\circ} \mathrm{C}$. ${ }^{\circ}$ Density of acid sites determined by $\mathrm{NH}_{3}$-TPD for $\mathrm{NH}_{3}$ desorbed at $180-280^{\circ} \mathrm{C}$.

Furthermore, FTIR results for the $\mathrm{OH}$ vibration region of zinccontaining and zinc-free conventional and desilicated ZSM-5 samples are shown in Figure 3. Generally, six bands with different intensities are observable, which are assigned as follows: 1) 3740 $\mathrm{cm}^{-1}$, isolated $\mathrm{Si}-\mathrm{OH}$ groups on the external surface; 2) $3723 \mathrm{~cm}^{-}$ 1 , isolated $\mathrm{Si}-\mathrm{OH}$ sites located inside the structure; 3) $3693 \mathrm{~cm}^{-1}$, weakly interacting $\mathrm{Si}-\mathrm{OH}$ sites located inside the structure; 4) $3664 \mathrm{~cm}^{-1}$, extra-framework Al-OH groups and possibly $\mathrm{Zn}-\mathrm{OH}$ groups; 5) $3606 \mathrm{~cm}^{-1}$, Brønsted acid sites of $\mathrm{Al}(\mathrm{OH}) \mathrm{Si}$ sites; 6) $3438 \mathrm{~cm}^{-1}$, strongly interacting $\mathrm{Si}-\mathrm{OH}$ groups which are referred to as silanol nests. ${ }^{[44]}$

Desilication, using the recrystallization approach, induces several changes in the concentration of different $\mathrm{OH}$ groups. The most apparent change is the formation of silanol nests, which goes hand in hand with an increasing amount of external and internal silanol groups. The desilication treatment apparently also generates some additional extra framework aluminum, which is obvious from the increasing intensities at $3664 \mathrm{~cm}^{-1}$ for all D-ZSM5 samples. Furthermore, the total concentration of Brønsted acid sites increases in D-ZSM- 5 sample due to selective extraction of $\mathrm{Si}$ from the conventional ZSM- 5 during the desilication. This is consistent with the measured concentration of strong acid sites by ammonia TPD. The intensity of $\mathrm{OH}$ vibration at $3606 \mathrm{~cm}^{-1}$, assigned to the Brønsted acid sites, decreases as the amount of zinc loading in C- and D-ZSM-5 increases, which is also in good agreement with the ammonia TPD results and previous studies. ${ }^{[3,7,45,46]}$ However, also some intensity decrease is observed for the bands at $3438,3693,3723$ and $3740 \mathrm{~cm}^{-1}$. It seems therefore that $\mathrm{Zn}$ is not only ion-exchanged at Brønsted 
acid sites, but also covering part of the external surface by bonding to the $\mathrm{Si}-\mathrm{OH}$ groups. Moreover, the intensity at $3664 \mathrm{~cm}^{-}$ 1 increases with the increase of zinc loading from 0.5 to $5 \mathrm{wt} \%$. This is caused due to formation of new Lewis acid sites created by incorporation of $\mathrm{Zn}$, as also reported in previous studies. ${ }^{[3,47]}$ In addition, the intensity of this peak is always higher in zinc containing D-ZSM-5 samples than the corresponding C-ZSM-5 samples, which is correlated to both slightly higher extra framework aluminum and new Lewis acid sites created by desilication and zinc incorporation, respectively. In general, it is clear that zinc is present as different species and located at various locations of zeolite.

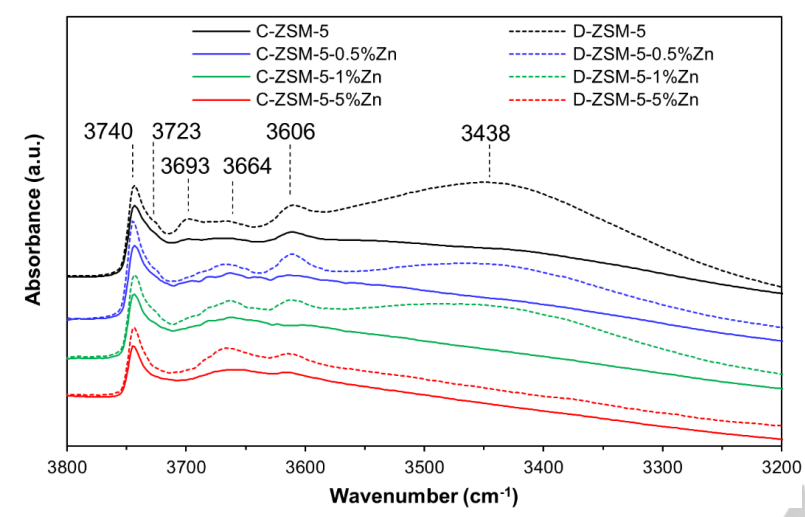

Figure 3. FTIR spectra in $v(\mathrm{OH})$ region of dehydrated zinc-containing conventional and desilicated ZSM-5 and their parent zeolites without zinc. All of the measurements were done at room temperature.

The ${ }^{29} \mathrm{Si}$ and ${ }^{27} \mathrm{AI}$ MAS NMR spectra were collected for C-ZSM-5, D-ZSM-5, C-ZSM-5-5\%Zn and D-ZSM-5-5\%Zn to determine the effect of desilication and zinc incorporation in the ZSM-5 zeolite samples (Figure 4). For all four samples, an intense peak centered at $54 \mathrm{ppm}$, assigned to tetrahedral framework aluminum and accounting for more than $95 \%$ of the total signal intensity dominates the ${ }^{27} \mathrm{Al}$ MAS NMR spectra. This confirms that the introduction of $Z n$ does not alter the local or long-range framework structure to any significant degree. For C-ZSM-5 and the desilicated analogue, a low-intense signal at $-1 \mathrm{ppm}$ is observed which can be assigned to extra-framework aluminum in octahedral coordination. No spinning-sidebands (ssb's) from the satellite transitions are observed in the spectra for the C-ZSM-5 and C-ZSM-5-5\%Zn samples, and thus the tetrahedral Al species exhibit a small quadrupolar coupling due to the highly symmetric environment. After desilication, ssb's are observed spanning at least $300 \mathrm{kHz}$. This reflects that the desilication process has introduced additional defects and disrupted the long-range order, which has resulted in distorted bond lengths and bond angles for the framework $\mathrm{AlO}_{4}$. This result is consistent with the observation of silanol nests and defects in the FTIR spectra.

From the ${ }^{29} \mathrm{Si}$ MAS NMR spectra it is possible to identify different tetrahedral silicate configurations with $\mathrm{Q}^{3}\left[\mathrm{Si}(\mathrm{OSi})_{3} \mathrm{OH}\right]$ around $\delta=$ $-103 \mathrm{ppm}, \mathrm{Q}^{4}(2 \mathrm{Al})$ and $\mathrm{Q}^{4}(1 \mathrm{Al})$ at $\delta=-107 \mathrm{ppm}$ and $\delta=-110 \mathrm{ppm}$, respectively, and $Q^{4}(0 \mathrm{Al})$ between -112 and $-117 \mathrm{ppm}$. Simulation of the total spectral line shape further allows for a semiquantitative determination of the different species. The Si/Al ratios determined from the peak areas are consistent with the Si/Al ratios as determined with ICP yielding Si/AI ratios of 39 and 35 for C-ZSM-5 and D-ZSM-5, respectively. It is well-known that the desilication process is selective towards $Q^{4}(0 \mathrm{Al})$ silicate species, and that the near-by presence of $\mathrm{AlO}_{4}$ tetrahedra in the framework structure shields the $Q^{4}(1 \mathrm{Al})$ and $Q^{4}(2 \mathrm{Al})$ sites, which is illustrated by the drop in Si/Al ratio for the desilicated samples. However, it is further observed by comparison of the ${ }^{29} \mathrm{Si}$ MAS NMR spectra that the desilication process also seems to be selective towards certain $\mathrm{Q}^{4}(\mathrm{OAl})$ sites, as the peak centered at $-114 \mathrm{ppm}$ is reduced to a larger degree than the peaks at $-112 \mathrm{ppm}$ and $-116 \mathrm{ppm}$. Overall, the $Q^{4}$-part of the ${ }^{29} \mathrm{Si}$ spectra are quite similar, consistent with the ${ }^{27} \mathrm{Al}$ NMR spectra showing that incorporation of zinc leaves the zeolite structure intact. From the peak simulations, the amount of $\mathrm{Q}^{3}-\mathrm{SiOH}$ are estimated to be $0.91 \%$ and $0.16 \%$ for $\mathrm{C}$ ZSM-5 and C-ZSM-5-5\%Zn, respectively. This observation further indicates that $\mathrm{Zn}-\mathrm{H}$ ion exchange is taking place. The desilication process increases the amounts of $\mathrm{Q}^{3}-\mathrm{SiOH}$ by a factor of 3 for both samples, most likely due to an increased amount of external surface $\mathrm{Si}-\mathrm{OH}$. The presence of $\mathrm{Zn}-\mathrm{OH}$ species in the CZSM-5-5\%Zn is confirmed with ${ }^{1} \mathrm{H}$ MAS NMR (Figure S2). Here a clear resonance is observed at a chemical shift of $1.25 \mathrm{ppm}$ for $\mathrm{C}$ ZSM-5-5\%Zn, which has previously been assigned to $\mathrm{Zn}-\mathrm{OH}$ moieties. ${ }^{[48]}$ The peak around $4.8 \mathrm{ppm}$ is rehydrated water, which masks the observation of Brønsted acid sites, typically observed around a chemical shift of $4.2 \mathrm{ppm}$. The signals between 1.6 and $2.1 \mathrm{ppm}$ can be assigned to silanol groups. ${ }^{[9]}$ However, it is noteworthy that zinc binding energy spectra from X-ray photoelectron spectroscopy (XPS) analysis did not indicate any significant difference between C-ZSM-5-5\%Zn and D-ZSM-5$5 \%$ Zn (Figure S6).

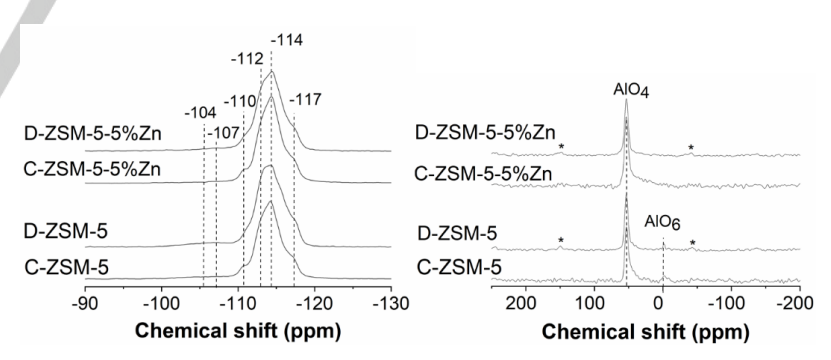

Figure 4. The ${ }^{29} \mathrm{Si}$ (left) and ${ }^{27} \mathrm{Al}$ (right) MAS NMR spectra for C-ZSM-5, D-ZSM$5, C-Z S M-5-5 \% Z n$ and D-ZSM-5-5\%Zn. The asterisks indicate spinning sidebands.

The conversion of ethane was followed over 30 hours of time on stream (TOS) at $550{ }^{\circ} \mathrm{C}, 2$ barg and GHSV $=0.3 \mathrm{~h}^{-1}$ for C-ZSM- 5 and D-ZSM-5 parent zeolites with $0.5,1$ and $5 \mathrm{wt} \%$ zinc, and is shown together with the BTX yields in Figure 5. The overall catalytic trend is that ethane conversion and BTX yield improve in all zinc-containing desilicated ZSM-5 catalysts. In addition, the results from ammonia TPD, FTIR and catalytic tests indicate that 
a higher $\mathrm{Zn}$ loading increases the number of Lewis active sites and catalytic activity.

Furthermore, the highest initial conversion is observed for DZSM- $5-5 \% Z n$, which is about $41 \%$, whereas, C-ZSM- $5-5 \% Z n$ shows $34 \%$ conversion. The same improvement in the conversion is observed for the other desilicated samples with 0.5 and $1 \mathrm{wt} \%$ zinc compared to conventional ones with the similar zinc loadings. However, although all catalysts are deactivating over time, the deactivation is significantly slower over the Zn-containing desilicated ZSM- 5 catalysts. For example, the conversion drops from $41 \%$ to $23 \%$ for D-ZSM-5-5\%Zn, while the decrease in conversion for C-ZSM-5-5\%Zn is from $34 \%$ to $10 \%$. The exact location of the deposited carbon has a significant influence on the deactivation mode of the catalyst. Therefore, it is speculated that coke formation starts blocking the pore entrances on the outer surface and pore intersections of microporous ZSM-5, which suffers from mass transfer limitation. As a result, active sites become less accessible due to carbon deposition in zinc containing conventional zeolites, whereas mesoporosity in the desilicated zeolites helps to facilitate mass transfer and increase the tolerance for coke deposition even though coke deposition occurs both internally and externally. ${ }^{[50,51]}$ Thus, the enhancement of catalytic performance in desilicated samples may be attributed to presence of mesoporosity and coke suppression.
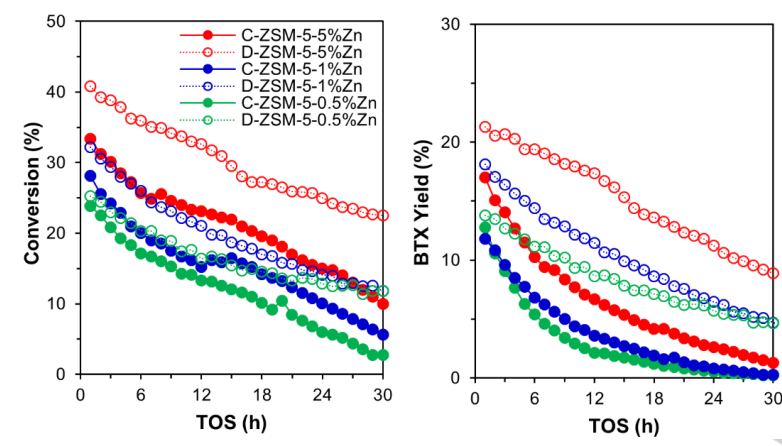

Figure 5. Conversion of ethane (left) and BTX yield (right) for zinc-containing CZSM-5 and D-ZSM- 5 catalysts over $30 \mathrm{~h}$ at $550^{\circ} \mathrm{C}, 2$ barg and $\mathrm{GHSV}=0.3 \mathrm{~h}^{-1}$.

As mentioned earlier, all of the desilicated ZSM- 5 zeolites loaded with zinc exhibit higher and more stable BTX yields than their respective conventional counterparts (Figure 5). Moreover, the highest yield of aromatics is observed at the highest conversion for all of the catalysts. It should also be noted that D-ZSM-5-5\%Zn has the highest initial yield of approximately $22 \%$ and the BTX yields are $18 \%$ and $14 \%$ for D-ZSM-5-1\%Zn and D-ZSM-5$0.5 \% \mathrm{Zn}$, respectively. After 30 hours of reaction, there is no BTX produced from ethane over the conventional ZSM- 5 with different zinc loadings, while the yield has reduced almost $50 \%$ in all $\mathrm{Zn}$ containing desilicated ZSM- 5 catalysts. This may confirm that despite coke formation, the active sites in the desilicated samples are still accessible for conversion of ethane to BTX.

Based on literature ${ }^{[6,7,15,26]}$, two types of zinc species are formed in a zinc-loaded ZSM-5. The spectator zinc, which is $\mathrm{ZnO}$ that can be easily reduced to metallic zinc with hydrogen, and the incorporated zinc that is hardly reduced. The presence of the incorporated active species of $\mathrm{ZnOH}^{+}$was confirmed with ${ }^{1} \mathrm{H}$ MAS NMR and FTIR. Generally, dehydroaromatization provides strongly reducing environment. Consequently, unreactive $\mathrm{ZnO}$ may be reduced to metallic zinc and leave the catalyst or some of reactive zinc species could anchor to the acid sites. ${ }^{[7,15,26]}$ Therefore, it is believed that the observed catalytic performance for all of the catalysts is the result of zinc, retained on active sites, strongly bound to the zeolite. Moreover, ICP-OES results of the spent catalysts (Table 2) are in good agreement with this assumption because in the catalysts with $5 \mathrm{wt} \%$ zinc loading, merely about one third of the zinc remains in the catalyst after 30 $\mathrm{h}$ TOS. However, the zinc content of samples with 0.5 and $1 \mathrm{wt} \%$ zinc seems to loose only a relatively small amount during the reaction. Therefore, it is likely that the spectator zinc species that leave the catalyst at early stage of the process have no role in the catalytic activity of the catalysts.

In order to investigate this assumption further, the two catalysts with 5 wt\% zinc (C-ZSM-5-5\% Zn and D-ZSM-5-5\%Zn) were regenerated after being on stream for 30 hours by heating under $1 \% \mathrm{O}_{2}$ in $\mathrm{N}_{2}$ at $600{ }^{\circ} \mathrm{C}$ for $2 \mathrm{~h}$. The results of activity test and selectivity towards BTX are plotted in Figure 6 . The test confirm that activity of the catalysts are retained and catalytic performances are similar to the fresh ones. This observation is consistent with the hypothesis that coke formation is the main reason for deactivation. In addition, it confirms that excess zinc
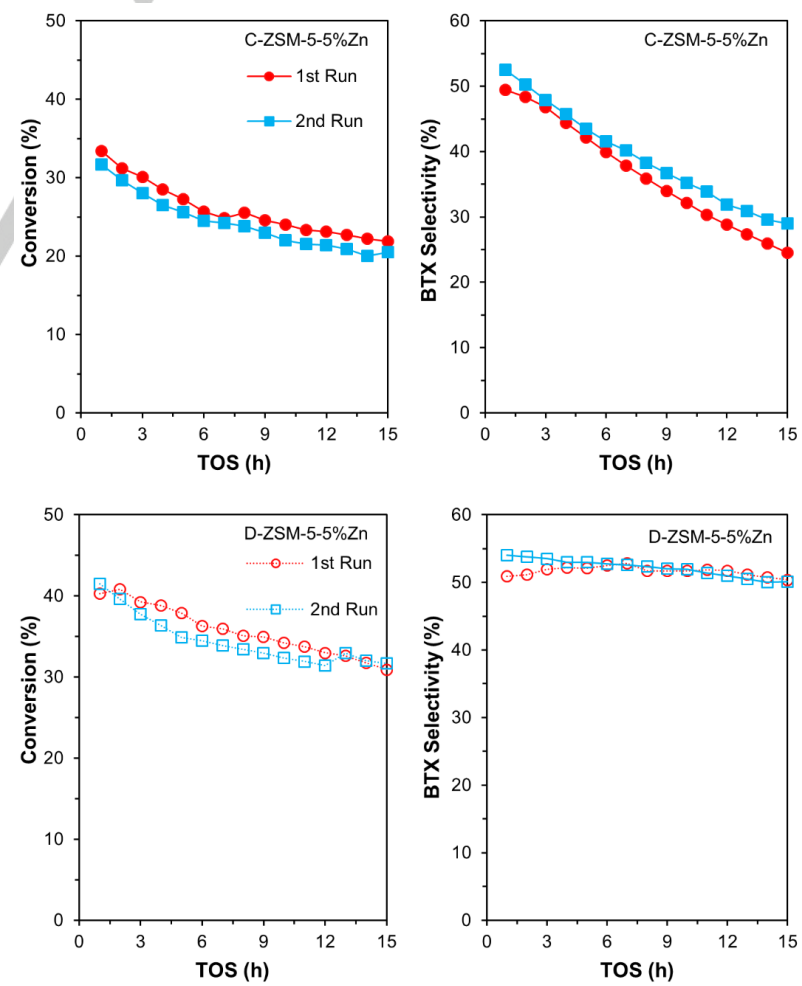

Figure 6. Catalytic results for the regenerated C-ZSM-5-5\%Zn and D-ZSM-5$5 \% \mathrm{Zn}$ over $15 \mathrm{~h}$ at $550{ }^{\circ} \mathrm{C}, 2$ barg and GHSV $=0.3 \mathrm{~h}^{-1}$. 
that is present as $\mathrm{ZnO}$ species in the fresh catalysts has no impact on catalytic reaction.

Selectivity towards methane and ethylene from ethane conversion over C-ZSM-5-5\%Zn and D-ZSM-5-5\%Zn, which have the highest conversion rate, is illustrated in Figure 7 . The selectivity to methane is proportional to the conversion implying that there is no transformation occurring for this alkane. The ethylene selectivity is higher in the C-ZSM-5-5\%Zn and the difference in selectivity for ethylene increases between these catalysts as the reaction proceeds. This indicates that in the conventional catalyst, further reaction of ethylene to produce aromatics is impeded due to inaccessibility of the acid sites in this catalyst caused by coking over time. Thus, ethylene selectivity varies from $17 \%$ to $80 \%$ for the C-ZSM-5-5\%Zn but it increases only from $7 \%$ to $34 \%$ for D-ZSM-5-5\%Zn after 30 hours of reaction.
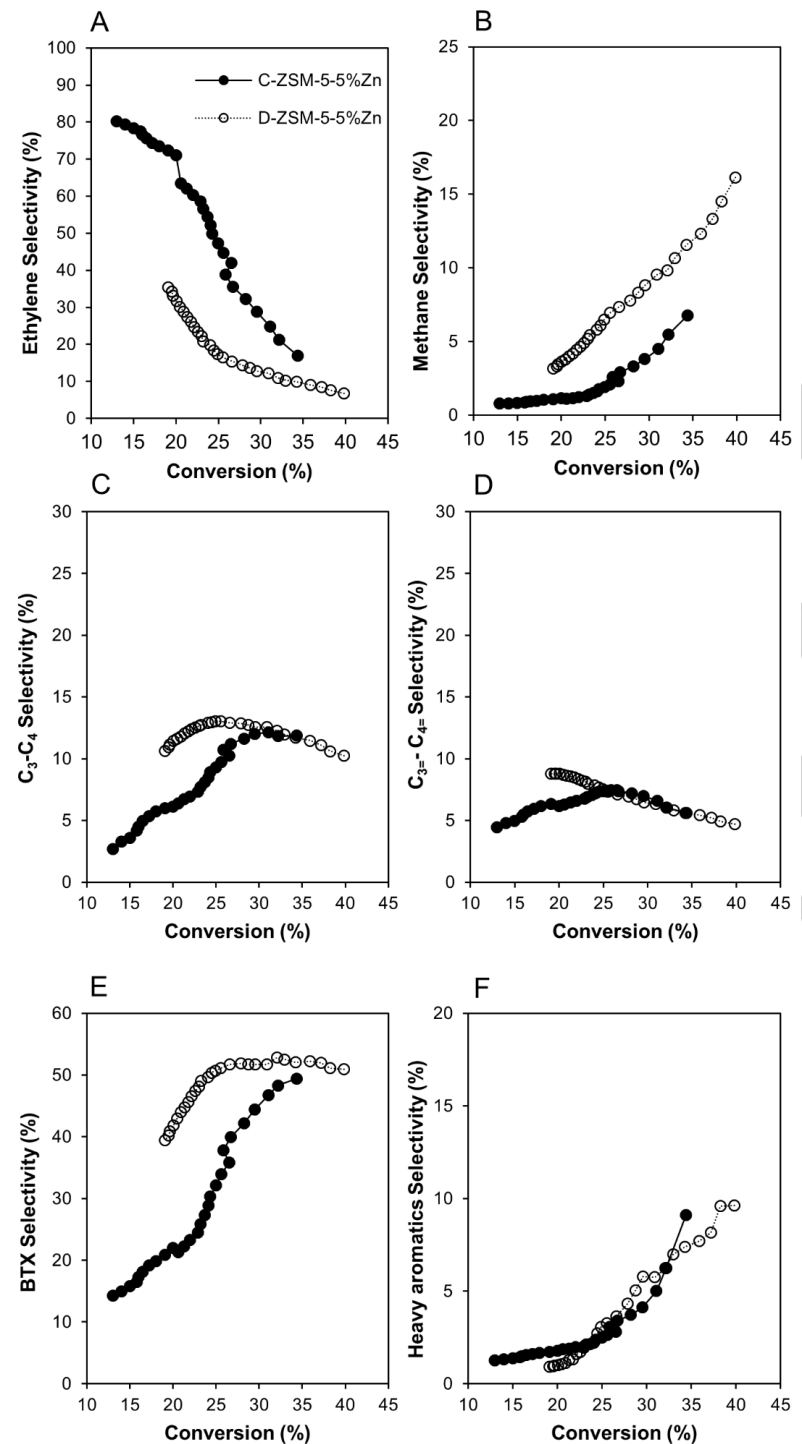

Figure 7. Selectivity to A) Ethylene, B) methane, C) alkanes $\left.\left(C_{3}-C_{4}\right), D\right)$ olefins $\left(\mathrm{C}_{3}=-\mathrm{C}_{4}=\right)$, E) BTX and $\mathrm{F}$ ) heavy aromatics products over $5 \mathrm{wt} \%$ zinc loaded conventional (filled spheres) and desilicated ZSM-5 (Empty spheres) at $550{ }^{\circ} \mathrm{C}, 2$ barg and $\mathrm{GHSV}=0.3 \mathrm{~h}^{-1}$.
The selectivity towards secondary products such as propane, butanes $\left(\mathrm{C}_{3}-\mathrm{C}_{4}\right)$, propene, butenes (olefins) and heavy aromatics including naphthalene derivatives (heavy aromatics), are also presented in Figure 7 . The $\mathrm{C}_{3}-\mathrm{C}_{4}$ and olefins selectivity over both catalysts passes a maximum and decreases, which indicates a secondary transformation of these products. Figure $7 \mathrm{~F}$ shows that formation of heavy aromatics such as naphthalene decreases for both catalysts as the conversion drops. Thus, it can be concluded that heavy aromatics are formed at the high conversion rate, in line with these compounds being secondary products. Furthermore, selectivity towards BTX is similar and only shows a modest dependence on the conversion for the D-ZSM-5-5\%Zn while it decreases substantially for the C-ZSM-5-5\%Zn catalyst depending on the conversion (Figure 7E). Moreover, the selectivity of each component in BTX selectivity is presented in Figure $S 4$, showing that there is no difference in benzene, toluene and xylenes distribution over C-ZSM-5-5\%Zn and D-ZSM-5$5 \% Z n$ catalysts.

All of the used catalysts were analyzed using TGA analysis to investigate the amount of deposited coke, which is the main reason behind the catalytic deactivation. As it is plotted in Figure 8 , there is a weight loss occurring due to water evaporation up to $300{ }^{\circ} \mathrm{C}$. The second weight loss is associated to burning off the coke. The differences in the coke contents seems to follow different trends for the samples with the same zinc loading. For instance, sample D-ZSM-5-0.5\%Zn barely shows weight loss due to less coke deposition and C-ZSM-5- $0.5 \%$ has $1 \mathrm{wt} \%$ coke content. The amount of coke deposited in the D-ZSM- $5-1 \% \mathrm{Zn}$ is insignificantly less than C-ZSM-5-1\%Zn. The amount of deposited coke increases in sample D-ZSM-5-5\%Zn to $3.9 \mathrm{wt} \%$ while it rises to 2.8 wt\% in C-ZSM-5-5\%Zn. This would suggest that D-ZSM-5$5 \% \mathrm{Zn}$ could accommodate more coke because of mesopores. Generally, deactivation of acidic zeolites such as ZSM- 5 catalysts is mainly caused by coking that results in blockage of micropore channels. ${ }^{[52,53]}$ Based on the catalytic results and coke contents, the presence of mesoporosity and voids in the D-ZSM- 5 zeolite facilitates diffusion of big molecules and coke precursors to diffuse out of the zeolite. Furthermore, mesopores in the framework would create an internal network of hierarchical porosity and provide better contact with the active sites under the circumstance of micropore blockage.

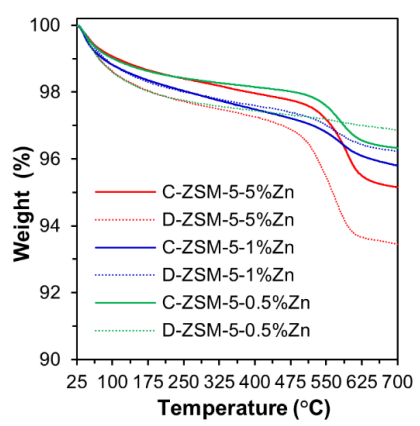

Figure 8. TGA analysis of the used catalysts with heating ramp of $5 \% \mathrm{~min}$ in synthetic air. 
In summary, $\mathrm{N}_{2}$ physisorption and TEM confirmed the presence of mesopores after desilication. FTIR and MAS NMR showed that selective desilication via recrystallization approach creates silanol nests and defects in zeolite framework. Moreover, ammonia TPD and FTIR results revealed similar observation to previous extensive investigations ${ }^{[3,7,45,46]}$, which was decrease in Brønsted acid sites and increase of Lewis acid sites concentration caused by zinc introduction to ZSM- 5 zeolites. Catalytic results showed that increasing the concentration of zinc improves the conversion and selectivity towards BTX. For comparison, Mehdad et al. ${ }^{[6]}$ tested $5 \mathrm{wt} \%$ zinc on ZSM- 5 catalyst with different Si/Al ratios and reported $20-50 \%$ drop in conversion at $500{ }^{\circ} \mathrm{C}$ after only $5 \mathrm{~h}$ of reaction while the activity only decreased by $10-25 \%$ in the desilicated Zn on ZSM- 5 samples at $550^{\circ} \mathrm{C}$ after $30 \mathrm{~h}$ of reaction. Furthermore, recycling the catalysts confirmed that $\mathrm{ZnO}$ leaves the catalyst during the reaction and has no catalytic activity. No dealumination of the zeolites were observed during recycling (see Figure S5 and Table S1). It should be pointed out that the zinc containing catalyst could be optimized by adjusting $\mathrm{Zn}$ to $\mathrm{Si} / \mathrm{Al}$ ratio. Furthermore, TGA results implied that created mesopores and voids improve the catalysts performance by decreasing the coke deposition and providing more accessibility to active sites.

\section{Conclusions}

We studied the influence of mesoporosity on catalytic performance of zinc-containing ZSM-5 catalysts in ethane dehydroaromatization. The formation of mesopores and voids in the microporous ZSM-5 zeolite was via selective desilication using ammonium hydroxide solution and in the presence of a surfactant. The desilicated zeolites were characterized and the results obtained from FTIR and MAS NMR showed that desilication leads to formation of silanol nests and defects due to removal of some silica and increase in strong acid sites density per gram of the zeolite. In addition, both FTIR and ammonia TPD confirmed that zinc incorporation decreases the amount of strong acid sites. The presence of $\mathrm{Zn}-\mathrm{OH}$ species were detected by ${ }^{1} \mathrm{H}$ MAS NMR and the formation of this specie results in appearance of new Lewis acid sites in FTIR spectra for all of the samples. Introducing mesopores via desilication, significantly improved the catalytic performance and BTX yield of Zn-containing ZSM-5 catalysts in direct conversion of ethane. In addition, increase of zinc content resulted in higher conversion and BTX yield. On the other hand, BTX selectivity was higher in the desilicated zeolites, which indicates that accessibility to the active sites governs the selectivity to the desired products. Regeneration of the catalysts showed that initial catalytic activity is regained upon burning off the coke, which confirms that the main reason of deactivation is carbon deposition and unavailability of the acid sites. Thus, desilicated zinc containing-ZSM-5 catalysts are promising in ethane dehydromatization process for providing longer catalytic lifetime and higher selectivity toward BTX products.

\section{Experimental Section}

Synthesis of conventional ZSM-5 (C-ZSM-5): $\sim 10 \mathrm{~g}$ of conventional ZSM-5 was synthesized from sodium aluminate $\left(\mathrm{NaAlO}_{2}\right.$, Sigma-Aldrich, anhydrous), tetrapropylammonium hydroxide (TPAOH, Sigma-Aldrich, $1.0 \mathrm{M}$ in $\mathrm{H}_{2} \mathrm{O}$ ) and tetraethyl orthosilicate (TEOS, Sigma-Aldrich, $\geq 99.0 \%$ ). In the synthesis procedure, $0.4 \mathrm{~g}$ sodium aluminate was dissolved in $72 \mathrm{ml}$ of TPAOH and then $44 \mathrm{ml}$ of TEOS was added dropwise while stirring in a Teflon beaker. After stirring for $1 \mathrm{~h}$, the mixture was transferred to a Teflon-lined stainless steel autoclave, heated at $180{ }^{\circ} \mathrm{C}$ and left for $24 \mathrm{~h}$. The product was filtered, washed with distilled water and dried at $80{ }^{\circ} \mathrm{C}$ overnight. Finally, the white powder was calcined for 20 hours at $550^{\circ} \mathrm{C}$ to achieve NaZSM5 . The sample was ion exchanged to the proton form by adding $10 \mathrm{~g}$ of NaZSM-5 in $800 \mathrm{ml}$ of $1 \mathrm{M}$ ammonium nitrate aqueous solution and stirring the solution at $80^{\circ} \mathrm{C}$ for $1 \mathrm{~h}$. The process was repeated 3 times. Afterwards, the solid was separated from the liquid, washed and dried for $12 \mathrm{~h}$ at $80^{\circ} \mathrm{C}$. The final product was calcined at $550^{\circ} \mathrm{C}$ for $10 \mathrm{~h}$ in air, designated as C-ZSM-5.

Synthesis of selectively desilicated ZSM-5 (D-ZSM-5): $10 \mathrm{~g}$ NaZSM-5 achieved from the synthesis procedure described above was added to a solution of $500 \mathrm{ml}$ ammonium hydroxide ( $15 \%$ ammonia in $\mathrm{H}_{2} \mathrm{O}$ ) and $7 \mathrm{~g}$ cetyltrimethylammonium bromide (CTAB) as surfactant and stirred for $3 \mathrm{~h}$ at room temperature. The mixture was then heated in a Teflon-lined stainless steel autoclave at $90^{\circ} \mathrm{C}$ for $24 \mathrm{~h}$ under autogenous pressure. Products were washed with distilled water and calcined for $10 \mathrm{~h}$ at $550^{\circ} \mathrm{C}$. The desilicated NaZSM- 5 was converted into its protonated form, following the aforementioned procedure for conventional ZSM-5, and designated as D-ZSM-5.

\section{Catalytic test}

Ethane dehydroaromatization experiments were performed in a continues fixed bed stainless steel reactor with inner diameter of $9 \mathrm{~mm}$ at the pressure of 2 barg and temperature of $550{ }^{\circ} \mathrm{C}$. Two thermocouples were placed in the reactor, one on the top and the other in the middle of the catalyst bed, to monitor temperatures at the inlet and in the catalyst bed, respectively. The inner surface of the reactor and the two thermowells that were in contact with the reactants were coated with an inert (SilcoNert ${ }^{8} 1000^{[54]}$ from Silicosteel $囚$, thickness of $100-500 \mathrm{~nm}$ ) to eliminate the effect of external interference on the conversion. Catalyst powder was pressed into pellets, crushed and sieved to fraction sizes of 0.6-1 $\mathrm{mm}$. In a typical run, $1.0 \mathrm{~g}$ of the fractionated catalyst was loaded in the reactor and pre-treated at $550{ }^{\circ} \mathrm{C}$ for $2 \mathrm{~h}$ under $12 \mathrm{NL} \mathrm{h}-1$ mixture flow of $\mathrm{N}_{2}$ with $1 \% \mathrm{O}_{2}$ to calcine zinc nitrate in the reactor. Subsequently, pure $\mathrm{N}_{2}$ flow purged for $1 \mathrm{~h}$ to remove any traces of oxygen. The reaction started by feeding the reactant gas (10\% ethane in $\mathrm{N}_{2}$ ) to the reactor, resulting in a GHSV of $0.3 \mathrm{NL} \mathrm{gcat}^{-1}$ $\mathrm{h}^{-1}$, and dehydroaromatization was carried out for $30 \mathrm{~h}$. The outlet gases were analyzed online in an Agilent 7890B gas chromatograph equipped with two capillary columns of CP. Al2O3-KCl $(25 \mathrm{~m} \times 0.32 \mathrm{~mm} \times 5 \mu \mathrm{m})$ and HP-INNOWax, $(30 \mathrm{~mm} \times 0.32 \mathrm{~mm} \times 0.25 \mu \mathrm{m})$, a thermal conductivity detector (TCD) and two flame ionization detectors (FID). The transfer line from the bottom of the reactor to the GC was heat traced to prevent condensation of products. Therefore, heavier products like naphthalene derivatives were also detected and quantified. The 
conversion and products selectivity were calculated based on moles of carbon. BTX yields are calculated by summing up benzene, toluene and xylenes yield. The operating temperature was selected based on initial reaction tests performed in an empty reactor as well as over conventional HZSM-5 at different temperatures, which showed that there was no reaction occurring at temperatures below $600{ }^{\circ} \mathrm{C}$ (supporting information Figure S3).

\section{Acknowledgements}

The authors are grateful for funding from Haldor Topsøe A/S, Independent Research Fund Denmark (grant no. 6111-00237), and Villum fonden (Grant No. 13158).

Keywords: heterogeneous catalysis $\cdot$ ethane $•$ dehydroaromatization $\cdot$ zeolites $\cdot$ aromatics

[1] M. N. Akhtar, N. Al-Yassir, S. Al-Khattaf, J. Čejka, Catal. Today 2012, 179, 61-72.

[2] Y. Jia, J. Wang, K. Zhang, W. Feng, S. Liu, C. Ding, P. Liu, Microporous Mesoporous Mater. 2017, 247, 103-115.

[3] I. P. Herrero, E. Borfecchia, J. Holzinger, U. V. Mentzel, F. Joensen, K. A. Lomachenko, S. Bordiga, C. Lamberti, G. Berlier, U. Olsbye, et al., J. Catal. 2018, 362, 146-163.

[4] G. Q. Zhang, T. Bai, T. F. Chen, W. T. Fan, X. Zhang, Ind. Eng. Chem. Res. 2014, 53, 14932-14940.

[5] A. Hagen, F. Roessner, Catal. Rev.: Sci. Eng. 2000, 42, 403-437.

[6] A. Mehdad, R. F. Lobo, Catal. Sci. Technol. 2017, 7, 3562-3572.

[7] X. Su, W. Zan, X. Bai, G. Wang, W. Wu, Catal. Sci. Technol. 2017,

$$
\text { 7, 1943-1952. }
$$

[8] Y. Xiang, H. Wang, J. Cheng, J. Matsubu, Catal. Sci. Technol. 2018, 8, 1500-1516.

[9] V. J. Frilette, P. B. Weisz, R. L. Golden, J. Catal. 1962, 1, 301-306.

[10] D. M. Nace, Ind. Eng. Chem. Prod. Res. Dev. 1969, 8, 31-38.

[11] N. Rahimi, R. Karimzadeh, Appl. Catal., A 2011, 398, 1-17.

[12] C. Zhang, G. Kwak, H. G. Park, K. W. Jun, Y. J. Lee, S. C. Kang, S. Kim, Microporous Mesoporous Mater. 2019, 276, 292-301.

[13] A. B. Laursen, K. T. Højholt, L. F. Lundegaard, S. B. Simonsen, S. Helveg, F. Schüth, M. Paul, J. D. Grunwaldt, S. Kegnœes, C. H. Christensen, et al., Angew. Chem., Int. Ed. 2010, 49, 3504-3507.

[14] J. Weitkamp, Solid State Ionics 2000, 131, 175-188.

[15] V. Abdelsayed, M. W. Smith, D. Shekhawat, Appl. Catal., A 2015, 505, 365-374.

[16] P. Schulz, M. Baerns, Appl. Catal., A 1991, 78, 15-29.

[17] Y. Ono, H. Kitagawa, Y. Sendoda, J. Jpn. Pet. Inst. 1987, 30, 7788.

[18] H. Wan, P. Chitta, J. Anal. Appl. Pyrolysis 2016, 121, 369-375.

[19] A. K. Rovik, A. Hagen, I. Schmidt, S. Dahl, I. Chorkendorff, C. H. Christensen, Catal. Letters 2006, 109, 153-156.

[20] K. Nishi, S. ichi Komai, K. Inagaki, A. Satsuma, T. Hattori, Appl. Catal., A 2002, 223, 187-193.

[21] N. Kosinov, F. J. A. G. Coumans, E. A. Uslamin, A. S. G. Wijpkema,

B. Mezari, E. J. M. Hensen, ACS Catal. 2017, 7, 520-529.

[22]

[23]

[24]

[25]

[26]

[27]

[28]

[29]

[30]

[30] M. Guisnet, P. Magnoux, D. Martin, in Stud. Surf. Sci. Catal., 1997, 111, 1-19.

[31] H. Saito, S. Inagaki, K. Kojima, Q. Han, T. Yabe, S. Ogo, Y. Kubota, Y. Sekine, Appl. Catal., A 2018, 549, 76-81.

[32] J. Čejka, G. Centi, J. P. Pariente, W. J. Roth, Catal. Today 2012, 179, 2-15.

[33] D. Verboekend, J. Pérez-Ramírez, Catal. Sci. Technol. 2011, 1, 879-890.

[34] I. I. Ivanova, E. E. Knyazeva, Chem. Soc. Rev. 2013, 42, 3671 3688.

[35] J. O. Abildstrøm, M. Kegnæs, G. Hytoft, J. Mielby, S. Kegnæs, Microporous Mesoporous Mater. 2016, 225, 232-237.

[36] A. F. Guzik, Microporous Mesoporous Mater. 2018, 259, 33-45.

[37] J. Mielby, J. O. Abildstrøm, S. P. Ferreras, S. B. Rasmussen, S. Kegnæs, J. Porous Mater. 2014, 21, 531-537.

[38] Y. Han, P. Pitukmanorom, L. Zhao, J. Y. Ying, Mesoporous Mater. 2012, 7, 326-332.

[39] J. Mielby, J. O. Abildstrøm, F. Wang, T. Kasama, C. Weidenthaler, S. Kegnæs, Angew. Chem., Int. Ed. 2014, 53, 12513-12516.

[40] F. Goodarzi, L. Kang, F. R. Wang, F. Joensen, S. Kegnæs, J. Mielby, ChemCatChem 2018, 10, 1566-1570.

[41] K. Egeblad, Microporous Mesoporous Mater. 2007, 101, 214-223.

[42] P. L. Llewellyn, J. P. Coulomb, Y. Grillet, J. Patarin, H. Lauter, H. Reichert, J. Rouquerol, Langmuir 1993, 9, 1846-1851.

[43] R. P. Thumbayil, J. Mielby, S. Kegnæs, Top. Catal. 2019, 62, 678688.

[44] M. S. Holm, S. Svelle, F. Joensen, P. Beato, C. H. Christensen, S. Bordiga, M. Bjørgen, Appl. Catal., A 2009, 356, 23-30.

[45] X. Chen, M. Dong, X. Niu, K. Wang, G. Chen, W. Fan, J. Wang, Z. Qin, Chin. J. Catal. 2015, 36, 880-888.

[46] X. Niu, J. Gao, Q. Miao, M. Dong, G. Wang, W. Fan, Z. Qin, J. Wang, Microporous Mesoporous Mater. 2014, 197, 252-261.

[47] V. B. Kazansky, V. Y. Borovkov, A. I. Serykh, R. A. Santen, B. G. Anderson, Catal. Lett. 2000, 66, 39-47.

[48] J.F. Wu, S.-M. Yu, W. D. Wang, Y.X. Fan, S. Bai, C.-W. Zhang, Q. Gao, J. Huang, W. Wang, J. Am. Chem. Soc. 2013, 135, 1356713573.

[49] M. Hunger, T. Horvath, J. Am. Chem. Soc. 1996, 118, 12302- 


\section{8.}

[50] F. Schmidt, C. Hoffmann, F. Giordanino, S. Bordiga, P. Simon, W. C. Cabrera, S. Kaskel, J. Catal. 2013, 307, 238-245.

[51] F. L. Bleken, K. Barbera, F. Bonino, U. Olsbye, K. P. Lillerud, S. Bordiga, P. Beato, T. V. W. Janssens, S. Svelle, J. Catal. 2013, 307, 62-73.

[52] M. S. Holm, E. Taarning, K. Egeblad, C. H. Christensen, Catal. Today 2011, 168, 3-16.

[53] J. C. Groen, W. Zhu, S. Brouwer, S. J. Huynink, F. Kapteijn, J. A. Moulijn, J. P. Ramírez, J. Am. Chem. Soc. 2007, 129, 355-360. "SilicoNert", can be found under https://silcotek.de/coating, 2019. 


\section{WILEY-VCH}

\section{FULL PAPER}

Entry for the Table of Contents (Please choose one layout)

Layout 1:

\section{FULL PAPER}

We selectively desilicated zinccontaining ZSM-5 via a simple method. Introduction of mesoporosity suppresses coke deposition and enhances catalytic life-time and selectivity to BTX for ethane dehydroaromatization at $550^{\circ} \mathrm{C}, 2$ barg and $\mathrm{GHSV}=0.3 \mathrm{~h}^{-1}$.

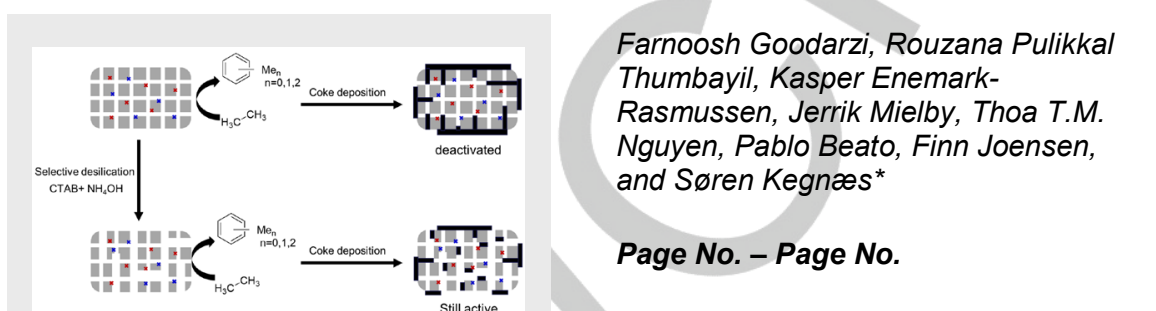

Zi:-itit Zeolite crystal

$x$ Bronsted acid sites

$\mathbf{x}$ Lewis acid stes
Enhanced catalytic performance of Zn-containing HZSM-5 upon selective desilication in ethane dehydroaromatization process 